\title{
Very deep spectroscopy of the bright Saturn nebula NGC 7009: The optical recombination spectrum and new effective recombination coefficients
}

\author{
Xuan Fang ${ }^{1}$, Xiao-Wei Liu ${ }^{1,2}$ and Peter J. Storey ${ }^{3}$ \\ ${ }^{1}$ Department of Astronomy, School of Physics, Peking University, Beijing 100871, P. R. China \\ email: fangx@pku.edu.cn \\ ${ }^{2}$ Kavli Institute for Astronomy and Astrophysics at Peking University, Beijing 100871, \\ P. R. China \\ ${ }^{3}$ Department of Physics and Astronomy, University College London, Gower Street, London \\ WC1E $6 \mathrm{BT}$, UK
}

\begin{abstract}
We present very deep spectroscopic observations of the rich optical recombination line (ORL) spectrum of the bright Saturn nebula NGC 7009. Detailed spectral analyses, combined with systematic emission line identifications, in the whole optical range (3040-11000 $)$ give more than 1000 emission lines, over $80 \%$ of which are ORLs. New calculations of the N II effective recombination coefficients in intermediate coupling scheme are carried out. These N II atomic data, together with the most recent $\mathrm{O}$ II and Ne II effective recombination coefficients, are utilized in the spectral analyses of NGC 7009. Departure from $L S$ coupling is noticed. Agreement is found between the observed and predicted relative intensities of ORLs. The elemental abundances of $\mathrm{C}, \mathrm{N}, \mathrm{O}$, and Ne deduced from ORLs are systematically higher than those from collisionally excited lines (CELs) by a factor of $5-7$.
\end{abstract}

Keywords. atomic data, atomic processes, line: identification, planetary nebulae: individual (NGC 7009)

\section{Observations and data reduction}

The spectra were observed by Liu from 1995 to 2001, using the ESO $1.52 \mathrm{~m}$ and WHT $4.2 \mathrm{~m}$ telescopes, and wavelength coverage is $3040-11100 \AA$, with a resolution (FWHM) of $1.5 \AA$ in the blue to about $3.0 \AA$ in the red. These are the deepest long-slit spectra ever taken for a planetary nebula $(\mathrm{PN})$. All the spectra were reduced with standard procedures using MIDAs. Due to the limited spectral resolution, multi-Gaussian profile fitting is used to obtain reliable fluxes of weak lines, especially the weak ORLs of C, N, O, and Ne ions. The flux uncertainties for the ORLs with most reliable measurements are less than $20 \%$, and for the strongest ones, less than $10 \%$. All emission features were further identified with an aid of the emission line identification code EMILI. In total there are more than 980 permitted lines identified, most of which are excited mainly by recombination, and about 230 CELs. The resulting emission line table for NGC 7009 is the most complete of all PNe ever studied.

\section{New effective recombination coefficients}

New calculations of the effective recombination coefficients for the $\mathrm{N}$ II recombination spectrum are carried out. We have taken into account the density dependence of the 


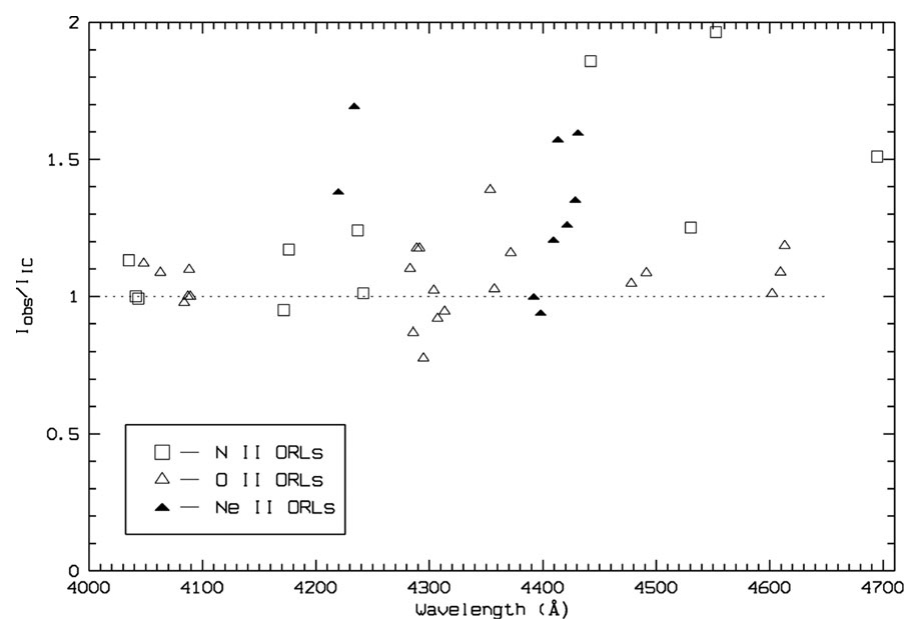

Figure 1. Comparison of the observed and predicted intensities of the N II (open squares), O II (open triangles), and Ne II ORLs (filled triangles). For the Ne II ORLs, only $4 \mathrm{f}-3 \mathrm{~d}$ transitions are presented, because the effective recombination coefficients for some of the strongest J-resolved transitions from the $4 \mathrm{f}-3 \mathrm{~d}$ configuration were calculated by P. J. Storey.

coefficients arising from the relative populations of the ground-term fine-structure levels of the recombining ion $\mathrm{N}$ III $\left({ }^{2} \mathrm{P}_{1 / 2}^{\mathrm{o}}\right.$ and $\left.{ }^{2} \mathrm{P}_{3 / 2}^{\mathrm{o}}\right)$, which opens up the possibility of electron density determination via recombination line analysis. Also new is the inclusion of dielectronic recombination at very low temperatures $(<250 \mathrm{~K})$. The new calculation is down to unprecedentedly low level $(\sim 100 \mathrm{~K})$. New effective recombination coefficients for the O II recombination spectrum were also calculated in intermediate coupling scheme by $\mathrm{P}$. J. Storey. The newly calculated effective recombination coefficients allow us to construct plasma diagnostics based on the reliable measurements of the $\mathrm{N}$ II and $\mathrm{O}$ II ORLs.

\section{Results of spectroscopic analysis}

$T_{\mathrm{e}}$ 's and $N_{\mathrm{e}}$ 's are derived from CELs (e.g., [O III], [N II], and [Ar IV] nebular and auroral lines), $\mathrm{H}$ I recombination spectrum, He I recombination lines, and $\mathrm{N}$ II and $\mathrm{O}$ II ORLs: $T_{\mathrm{e}}(\mathrm{CELs}) \approx 10^{4} \mathrm{~K}, T_{\mathrm{e}}(\mathrm{H} \mathrm{I} \mathrm{BJ})=6500 \mathrm{~K}, T_{\mathrm{e}}(\mathrm{He} \mathrm{I})=5100 \mathrm{~K}, T_{\mathrm{e}}(\mathrm{ORLs}) \approx 1000 \mathrm{~K}$. Thus the general pattern of electron temperatures, $T_{\mathrm{e}}(\mathrm{CELs}) \gtrsim T_{\mathrm{e}}(\mathrm{H} \mathrm{I} \mathrm{BJ}) \gtrsim T_{\mathrm{e}}(\mathrm{He} \mathrm{I})$ $\gtrsim T_{\mathrm{e}}$ (ORLs), which is seen in many PNe, is repeated in NGC 7009. This supports the possibility of the nebular bi-abundance model (see the contribution of Liu in this volume and references therein).

We analyzed the ORLs with most reliable measurements, utilizing the new effective recombination coefficients. Agreement is found between the observed and predicted relative intensities of N II, O II, and Ne II ORLs. Figure 1 shows the ratios of the observed $\left(I_{\text {obs }}\right)$ and predicted intensities $\left(I_{\mathrm{IC}}\right)$ of $\mathrm{N}$ II, O II, and Ne II ORLs with most reliable measurements (errors < 20\%).

The ionic abundances derived from ORLs are all higher than those from the IR, optical, and UV CELs. The $\mathrm{N}^{2+} / \mathrm{H}^{+}$and $\mathrm{O}^{2+} / \mathrm{H}^{+}$abundance ratios derived from different ORLs agree with each other, while $\mathrm{Ne}^{2+} / \mathrm{H}^{+}$ratios show relatively large scattering, and the average $\mathrm{Ne}^{2+} / \mathrm{H}^{+}$value from $3-3$ transitions are lower than that from $4 \mathrm{f}-3 \mathrm{~d}$ by 0.15 dex, indicating that more systematic and accurate calculations of the Ne II effective recombination coefficients are needed. The C, N, O, and Ne elemental abundances yielded by ORLs are higher than those from CELs by a factor of $5-7$. 